Full length article

\title{
Cyclostationary signatures for LTE Advanced and beyond
}

\author{
P.D. Sutton ${ }^{\mathrm{a}, *}$, B. Özgül ${ }^{\mathrm{b}, 1}$, L.E. Doyle ${ }^{\mathrm{a}}$

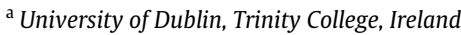 \\ ${ }^{\mathrm{b}}$ Xilinx, Dublin, Ireland
}

\section{A R T I C L E I N F O}

\section{Article history:}

Received 6 July 2011

Accepted 4 November 2013

Available online $\mathrm{xxxx}$

\section{Keywords:}

Cyclostationary signatures

LTE Advanced

Network coordination

Rendezvous

OFDMA

Carrier aggregation

Dynamic spectrum access

\begin{abstract}
A B S T R A C T
Long Term Evolution (LTE) Advanced is the next generation of the LTE standard, offering peak data rates of up to $1 \mathrm{Gbps}$ using up to $100 \mathrm{MHz}$ of spectrum. A key mechanism in achieving this is carrier aggregation (CA) whereby multiple LTE component carriers (CCs) are combined in a contiguous or non-contiguous fashion. With the introduction of $C A$ comes the challenge of network rendezvous. In order to associate with an LTE Advanced basestation or eNodeB, a User Equipment (UE) device must be capable of detecting component carriers in use by that eNodeB and establishing communications links. Looking beyond LTE Advanced, the introduction of service and technology neutral approaches to spectrum management is likely to increase the importance of rendezvous, as more diverse spectrum bands become available for use. This paper presents cyclostationary signatures as a powerful tool for overcoming the challenge of network rendezvous in LTE Advanced networks and beyond. A signature detector design, based on the Autocoherence Function $(\mathrm{AF})$, is presented and a number of mechanisms for embedding signatures in downlink LTE CC waveforms are described. The performance of our signature detector is examined in depth through simulation under conditions of doubly-selective fading. Simulation results highlight the performance advantages which can be achieved through use of the AFbased detector over the simpler time-smoothed cyclic cross periodogram (TS-CCP)-based detector. Over the air experiments using a software radio based transceiver are described and results are presented.
\end{abstract}

(C) 2013 Elsevier B.V. All rights reserved.

\section{Introduction}

In order to meet the performance and technical requirements specified for fourth generation (4G) mobile systems, Long Term Evolution (LTE) Advanced standards offer improvements over preceding LTE standards, while supporting backward compatibility. Carrier aggregation (CA) is one of the key improvements in LTE Advanced, which allows the aggregation of two or more LTE carriers to increase the bandwidth for a single user equipment (UE) device up to $100 \mathrm{MHz}$ [1]. Each individual carrier

\footnotetext{
* Corresponding author.

E-mail addresses: suttonpd@tcd.ie (P.D. Sutton),

baris.ozgul@gmail.com (B. Özgül), linda.doyle@tcd.ie (L.E. Doyle).

${ }^{1}$ B. Özgül contributed to this work while working with Trinity College.
}

is called a component carrier (CC), which can support one of the bandwidth configurations ranging from 1.4 to $20 \mathrm{MHz}$. LTE Advanced allows both intra- and inter-band CA, where the CCs are located in the same band or different bands, respectively. In both cases, CCs aggregated within a band can be contiguous or non-contiguous. Despite its benefits, CA poses a number of technical challenges in practice. These challenges include the flexible use of a wide range of frequency bands, fast and robust detection of the CCs involved in CA by the UEs, and the design of control signalling, while being backward compatible with LTE systems. In this paper, we apply a tested mechanism for network coordination in dynamic spectrum access systems to the challenge of CA in LTE Advanced systems and beyond.

Initial specifications for LTE Advanced have identified a limited number of frequency bands for intra- and inter- 
band CA [2]. However, progression beyond LTE Advanced and the consideration of paradigms such as the opening up of the TV white spaces to secondary use [3] and the introduction of technology and service neutral use of the radio spectrum [4] promote the use of CA over a much wider range of frequency bands.

One of the biggest challenges in this case is network rendezvous, which requires the detection of individual CCs by the LTE Advanced UEs and establishment of the communications links on those carriers. In this paper, we propose embedding robust features into downlink frames on each CC to facilitate successful network rendezvous, at the expense of introducing only a small level of overhead in the downlink transmissions. These intentionally embedded features are called cyclostationary signatures. Previous work has shown that their use provides a powerful tool for achieving network rendezvous and coordination in reconfigurable wireless networks using multi-carrier waveforms, without any need for a static common control channel. Signatures can be used for signal detection, identification, carrier frequency acquisition and bandwidth estimation [5-9].

Our first contribution in this paper is to show how cyclostationary signatures can be embedded into LTE downlink frames. We introduce two different approaches. The first approach is fully compatible with the LTE physical downlink frame structure and requires minor changes in the LTE basestation (eNodeB) scheduler, whereas the latter approach results in some specific changes in the eNodeB scheduler and transmitter and the UE receivers but incurs less overhead. We describe a signature detector, based on the autocoherence function (AF), and present new simulation and experimental results to examine its performance under doubly-selective fading channels.

The remainder of the paper is organized as follows. Section 2 provides background information on cyclostationary signatures and their generation and presents the AF-based detector. The use of cyclostationary signatures to facilitate CA in LTE Advanced and beyond is addressed in Section 3. Simulation and experimental results are presented in Section 4 and the conclusions can be found in Section 5 .

\section{Cyclostationary signatures}

A signal is cyclostationary if there exists some nonlinear transformation of that signal which will generate finitestrength additive sine-wave components [10]. A signal is said to exhibit second-order cyclostationarity if its mean and autocorrelation are periodic.

Many of the communications signals in use today exhibit second and higher-order cyclostationarity due to underlying periodicities introduced through coupling stationary message signals with periodic sinusoidal carriers, pilot sequences, spreading codes and repeating preambles. It has been shown that these cyclostationary properties can be used to achieve a number of critical tasks including signal detection [11], classification [12], synchronization $[13,14]$ and equalization [15].

Cyclostationary signatures are features which are not inherent to a signal of interest but are rather intentionally embedded for a particular purpose. It has been shown

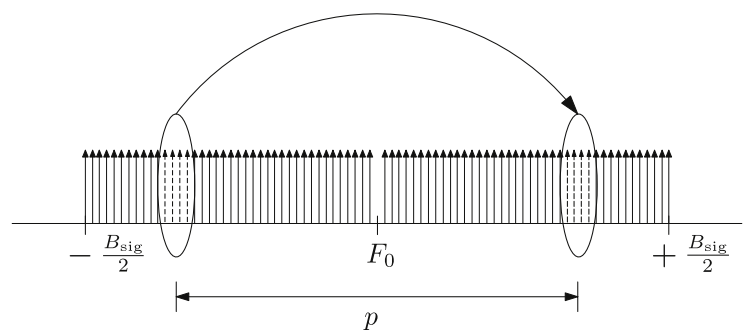

Fig. 1. Generation of a cyclostationary signature using OFDM subcarrier set mapping.

that these signatures or watermarks provide an effective tool for achieving rendezvous and network coordination in dynamic spectrum access networks [7]. The key advantage of using embedded cyclostationary features is that they form a very low-level physical property of the signal. As such, they may be detected and analysed prior to time or frequency synchronization and with very little prior knowledge about the physical parameters of the signal.

Intentionally embedded features provide a number of key advantages over the use of inherent signal features. Firstly, embedded features can be easily manipulated to suit the requirements of the system designer. With inherent features this is not typically possible without significantly changing the physical properties of the signal in question. Secondly, embedded features can be easily generated in the signal of interest and can be detected and analysed using low-complexity detector designs. Furthermore, embedded features can typically be detected using shorter observation times than those required for reliable detection of inherent features.

\subsection{Signature generation}

Cyclostationary signatures can be easily embedded in multicarrier waveforms through subcarrier mapping. This process involves the transmission of identical data symbols on two discrete sets of subcarriers and is illustrated in Fig. 1 for an Orthogonal Frequency Division Multiplex (OFDM) signal. Here, $F_{0}$ is the DC carrier, $B_{\text {sig }}$ is the signal bandwidth and $p$ is the subcarrier set separation.

OFDM signals may be represented as a composite of $N$ statistically independent subchannel Quadrature Amplitude Modulated (QAM) signals [16]:

$w(t)=\sum_{k} \sum_{n=0}^{N-1} \gamma_{n, k} e^{j\left(2 \pi / T_{S}\right) n t} q(t-k T)$

where $w(t)$ is the complex envelope of an OFDM signal with a cyclic prefix, $\gamma_{n, k}$ is the independent, identically distributed message symbol transmitted on subcarrier $n$ during OFDM symbol $k, N$ is the number of subcarriers and $q(t)$ is a square shaping pulse of duration $T . T_{S}$ is the source symbol length and $T_{g}$ is the cyclic prefix length such that $T=T_{s}+T_{g}$.

Subcarrier set mapping is carried out as:

$\gamma_{n, k}=\gamma_{n+p, k}, \quad n \in M$

where $M$ is the set of subcarrier values to be mapped and $p$ is the number of subcarriers between mapped symbols. 


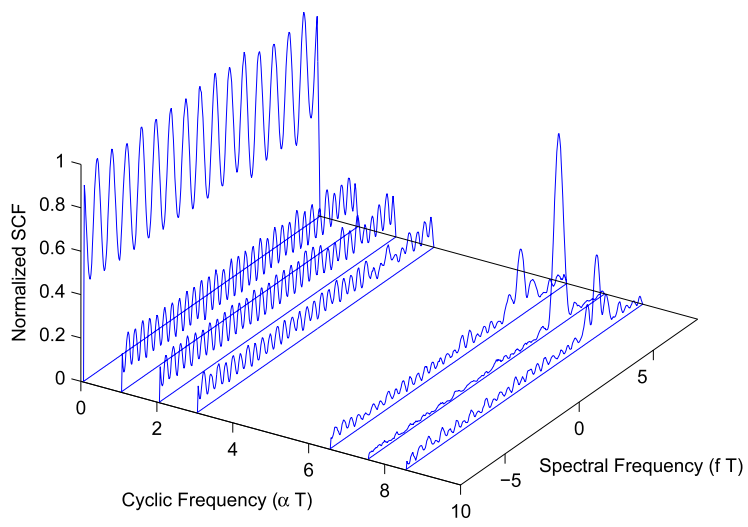

Fig. 2. Normalized SCF for OFDM with cyclic prefix and embedded cyclostationary signature.

Fig. 2 illustrates the Spectrum Correlation Function (SCF) for an OFDM signal generated in this way. In this case 16 subcarriers are used and a single subcarrier is mapped with subcarrier set separation $p=6$. The cyclic prefix in this case is $1 / 4$ of the OFDM symbol length. The resulting feature can be clearly seen at cyclic frequency $\alpha T=7.5$ where $T$ is the symbol length.

\subsection{Signature detection}

A single cycle signature detector can be implemented using the time-smoothed cyclic cross periodogram (TSCCP) [17]:

$\hat{S}_{x}^{\alpha}[k]=\frac{1}{L} \sum_{l=0}^{L-1} X_{l}[k] X_{l}^{*}[k-\alpha] W[k]$

where $W[k]$ denotes a smoothing spectral window and $X_{l}[k]$ is the discrete Fourier transform of the $l$ th window of received signal samples, $x_{l}[n]$,

$X_{l}[k]=\sum_{n=0}^{N-1} x_{l}[n] \exp ^{\frac{-j 2 \pi n k}{N}}$

Estimates are calculated using $L$ windows of length $N$ where $N$ is the duration of a single OFDM symbol in discrete time.

The TS-CCP can be used directly to perform signature detection. However, improved performance can be achieved through power normalization using the Autocoherence Function AF [18]:

$\hat{C}_{x}^{\alpha}[k]=\frac{\hat{S}_{x}^{\alpha}[k]}{\left(\hat{S}_{x}^{0}[k] \hat{S}_{x}^{0}[k-\alpha]\right)^{1 / 2}}$.

Cyclostationary features generated by OFDM subcarrier set mapping may be successfully detected using spectral resolution $\Delta f$, equal to the OFDM subcarrier spacing. Using this approach, the ideal SCF may be approximated using a simple rectangular window of width $M . \Delta f$ [11], where $M$ is the number of subcarriers in the mapped set. In this way,

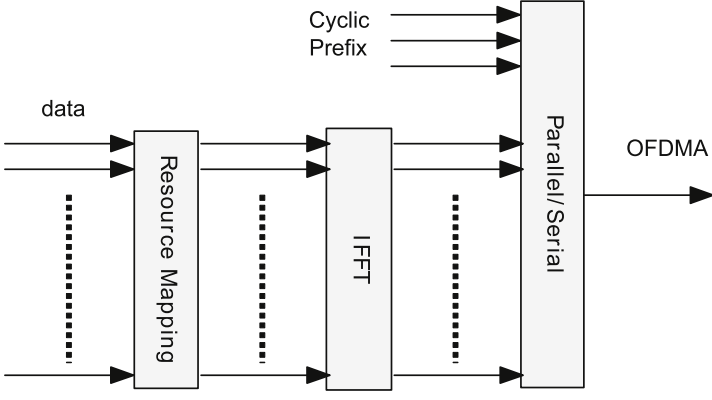

Fig. 3. Generation of LTE downlink OFDMA waveform.

a low-complexity single-cycle signature detector may be implemented as:

$y_{\alpha}=\max _{0 \leq k \leq N-1}\left|\sum_{m=0}^{M-1} R[m] \hat{C}_{x}^{\alpha}[k-m]\right|$

where $R[m]$ is a rectangular window of length $M$.

Prior work by the authors has examined the performance of a TS-CCP based signature detector [7]. Section 4 presents a wide range of simulation and experimental results obtained using the more recent AF based detector, illustrating the significant reduction in required observation time that can be achieved.

\section{Signatures for LTE Advanced and beyond}

As discussed in Section 1, network rendezvous is a key challenge faced in progressing from Long Term Evolution (LTE) to LTE Advanced. This challenge arises due to the use of carrier aggregation, required to achieve the target peak data rate of $1 \mathrm{~Gb} / \mathrm{s}$ specified for future IMT-Advanced mobile systems [19].

In order to reach such high data rates, it is necessary for IMT-Advanced systems to operate using up to $100 \mathrm{MHz}$ of spectrum. Operators are unlikely to be successful in accessing such bandwidth in a single continuous band at frequencies suitable for non-line of sight transmission. Therefore, individual non-continuous LTE carriers, each of up to $20 \mathrm{MHz}$, must be aggregated across one or more spectrum bands. Thus LTE User Equipment (UE) devices must be capable of detecting individual LTE carriers being used by nearby LTE Base-Stations (eNodeBs) and establishing communication links on those carriers. This is the challenge of network rendezvous.

This paper proposes the use of intentionally embedded cyclostationary signatures to address the challenge of network rendezvous for LTE Advanced and presents new simulation and experimental results, achieved using a novel AF based signature detector.

In order to use cyclostationary signatures in LTE Advanced, one approach would be for eNodeBs in the network to embed one or more features in all downlink transmissions. This can be done at the resource mapping stage of the eNodeB architecture, just prior to the Inverse Fast Fourier Transform (IFFT) stage (see Fig. 3).

In this way, each downlink transmission would contain a signature which could be detected by UEs devices, used 


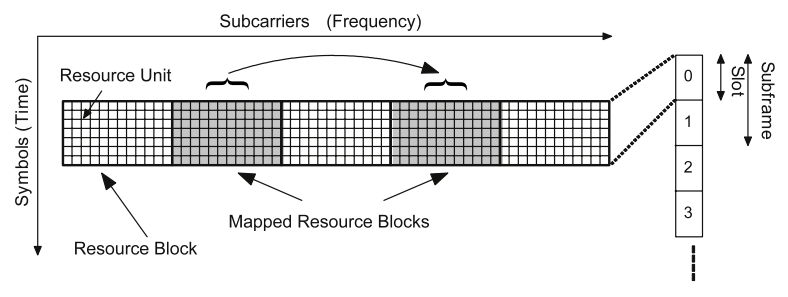

Fig. 4. Signature generation through resource block mapping.

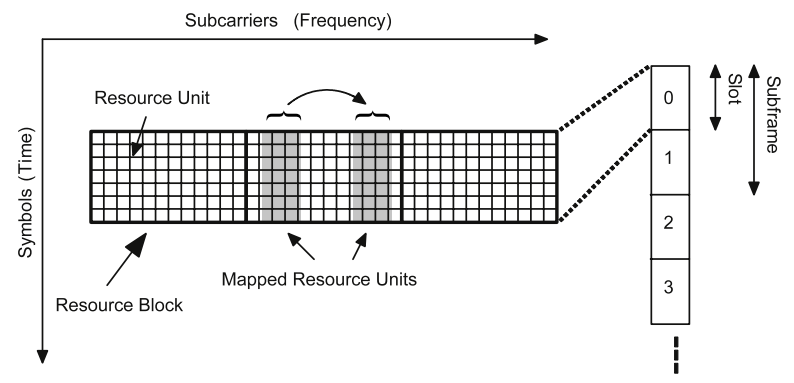

Fig. 5. Signature generation through resource unit mapping.

to identify the eNodeB and LTE carrier and used to achieve synchronization with that carrier before establishing a communications link.

A signature could be embedded in a downlink transmission by an eNodeB in one of two ways. The first approach is to map a full LTE resource block onto another resource block as shown in Fig. 4. The advantage of this approach is that minor changes would be required in the transmitter architecture. As resource blocks are independently scheduled, the process of mapping could be carried out in a straightforward manner by the eNodeB scheduler. A resource block consists of 12 subcarriers, so this approach would result in a powerful signature but would also incur a relatively high overhead. Standard UE devices are also unaffected by this approach as the eNodeB scheduler can simply choose not to assign mapped resource blocks to any UE.

A second approach which would be more efficient would be to map small numbers of subcarriers within individual resource blocks as shown in Fig. 5. This approach would incur less overhead but would involve more complex changes to the transmitter architecture. In addition, this approach requires UE devices to be informed as to which resource units are mapped in which blocks so that they can successfully demodulate the data transmitted in those blocks. This can be achieved through prior specification of mapped resources or through the use of additional control information transmitted by the eNodeB.

Adopting either approach for embedding signatures in downlink transmissions involves a level of overhead as mapped resources cannot be used to carry independent data. However, by mapping a resource, we provide redundancy for the data carried on that resource. This redundancy may allow us to adopt a higher modulation order or a reduced coding rate, thus lessening the overhead incurred.
In generating our embedded signatures, reference signals are not mapped.

The use of resource mapping to generate signatures means that those signatures are present at all times in downlink transmissions. In Frequency Division Duplex (FDD) LTE, a UE can detect an embedded signature by analysing any portion of the downlink transmission. However, in Time Division Duplex (TDD) LTE, downlink and uplink transmissions are multiplexed on the same frequency channel. In this case, signatures are only present during downlink bursts and a different approach may be needed for UEs to detect them.

In order to detect signatures in bursty transmissions, an iterative detector can be employed. In this way UEs can monitor a channel for signature-containing transmissions using a dwell time which is longer than the observation time of the detector.

The iterative detector uses the AF as before in Eq. (5) but iteratively calculates the TS-CCP:

$$
\begin{aligned}
\hat{S}_{x}^{\alpha}[k]_{\ell+1}= & \hat{S}_{x}^{\alpha}[k]_{\ell}+X_{\ell+L}[k] X_{\ell+L}^{*}[k-\alpha] W[k] \\
& -X_{\ell}[k] X_{\ell}^{*}[k-\alpha] W[k]
\end{aligned}
$$

where $\hat{S}_{x}^{\alpha}[k]_{0}=\hat{S}_{x}^{\alpha}[k], X_{\ell}[k]$ is the discrete Fourier transform of $x_{\ell}[n], 0 \leq \ell \leq\left(L_{U}+L\right)$, $L$ is the number of observation windows considered by the detector at each iteration, and $L_{U}$ is the LTE uplink frame duration in terms of observation windows. It is assumed that $L$ is less than or equal to the downlink frame length. The AF at the $\ell$ th iteration, $\hat{C}_{x}^{\alpha}[k]_{\ell}$, is found by inserting $\hat{S}_{x}^{\alpha}[k]_{\ell}$ into (5). Then, the decision metric at the lth iteration, $y_{\alpha, \ell}$, is computed by using $\hat{C}_{x}^{\alpha}[k]_{\ell}$ in (6). The final decision metric is found as:

$y_{\alpha}=\max _{0 \leq \ell \leq\left(L+L_{U}\right)} y_{\alpha, \ell}$.

Using (8), the iterative detector does not need any time synchronization with the downlink frames for robust decisions.

\section{Performance}

\subsection{Simulation}

In generating a cyclostationary signature using OFDM subcarrier set mapping, a significant trade off exists between the number of subcarriers used to embed the signature and the detection performance which may be achieved. Increasing the mapped set size results in improved performance. However, as mapped subcarriers cannot transmit independent data symbols, this results in reduced overall capacity.

The performance of signatures created using different mapped subcarrier set sizes is examined using simulation. 256-subcarrier OFDM signals are considered with carriers designated as follows: 192 data, 8 pilot, 55 guard, 1 DC (zero-frequency) carrier. Subcarriers are modulated using Quadrature Phase Shift Keying (QPSK) message symbols and a $1 / 16$ cyclic prefix is adopted. Signatures with an arbitrarily chosen cyclic frequency, $\alpha=32 / T_{\mathrm{s}}$ are generated using subcarrier set mapping. Gaussian white noise is added to each signal to result in SNR values of between 


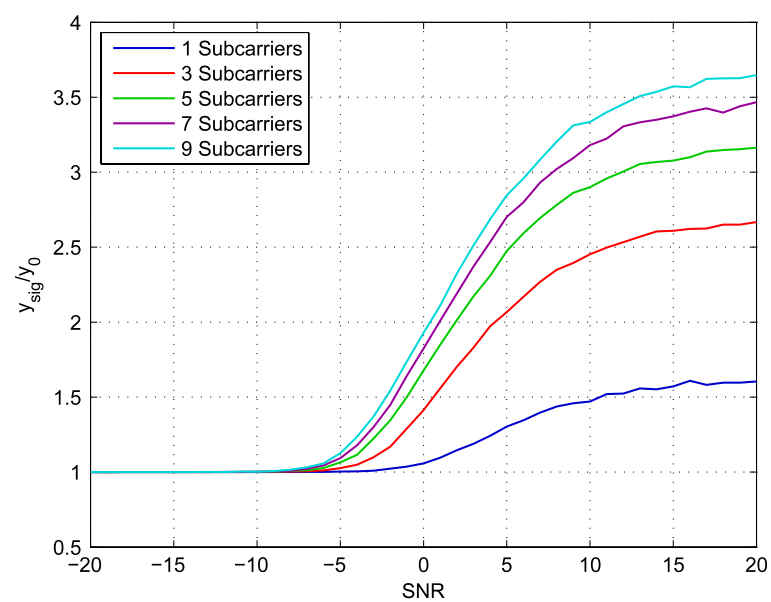

Fig. 6. Signature detection ratio performance with increasing mapped subcarrier set sizes over a range of SNR values.

-20 and $20 \mathrm{~dB}$. In addition a random timing offset is added according to a uniform distribution over a single OFDM symbol. Detection statistics are recorded for signals containing embedded signatures $\left(y_{\text {sig }}\right)$ and those in which subcarrier set mapping is not used $\left(y_{0}\right) .1000$ simulations are carried out for each and mean values are used to generate the detection ratio $y_{\text {sig }} / y_{0}$. This ratio illustrates the distance between mean detection statistics for signals containing signatures and those without embedded features and may be interpreted as a measure of the confidence with which detection decisions can be made.

Results are illustrated in Fig. 6 for observation time, $\Delta t=30 T$ where $T$ is the OFDM symbol duration.

As expected, results illustrate the improvement in detection performance associated with increasing the size of the OFDM subcarrier sets used to embed signatures.

It can be seen that detector performance deteriorates rapidly with SNR $<0 \mathrm{~dB}$, to the stage where $y_{\text {sig }}$ and $y_{0}$ are indistinguishable at SNR $<-5 \mathrm{~dB}$. This suggests that cyclostationary signatures may not be used successfully to facilitate very low-power signal detection when short signal observation times are adopted. However, in the context of LTE carrier detection and rendezvous, UE devices must synchronize with and successfully demodulate detected signals. At SNR levels below which detection may be performed OFDM based systems typically experience very high bit-error rates and rendezvous cannot be achieved [20].

A key performance metric for cyclostationary signatures used in the context of rendezvous and coordination is the time taken to reliably detect and analyse an embedded signature. Although reliable analysis of inherent signal features typically requires high spectral resolution and long signal observation times, the use of cyclostationary signatures facilitates the use of spectral resolution on the order of OFDM subcarrier spacings and thus relatively short observation times.

The effect of observation times upon signature detection performance is examined using further simulations. 256-subcarrier OFDM signals are considered as before. Signatures are embedded using mapped subcarrier set sizes of

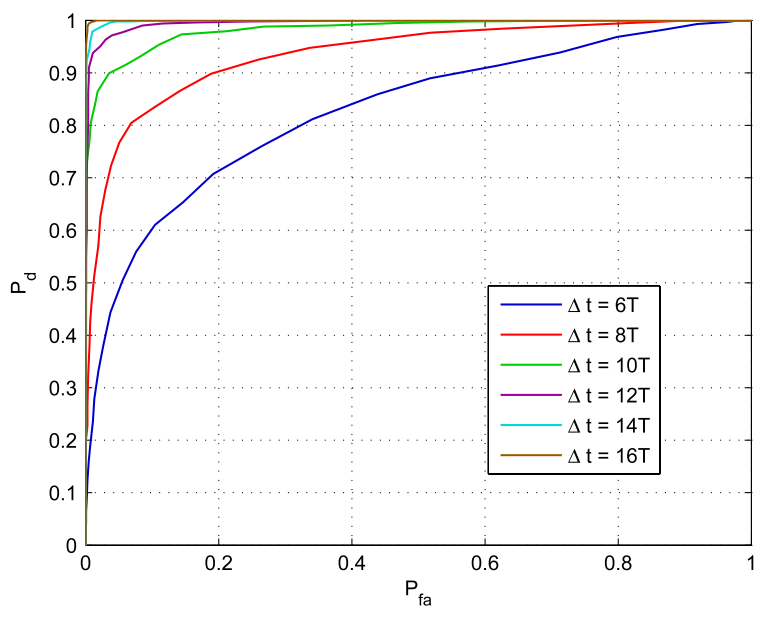

Fig. 7. Receiver Operating Characteristic (ROC) performance with increasing observation time for $M=3$.

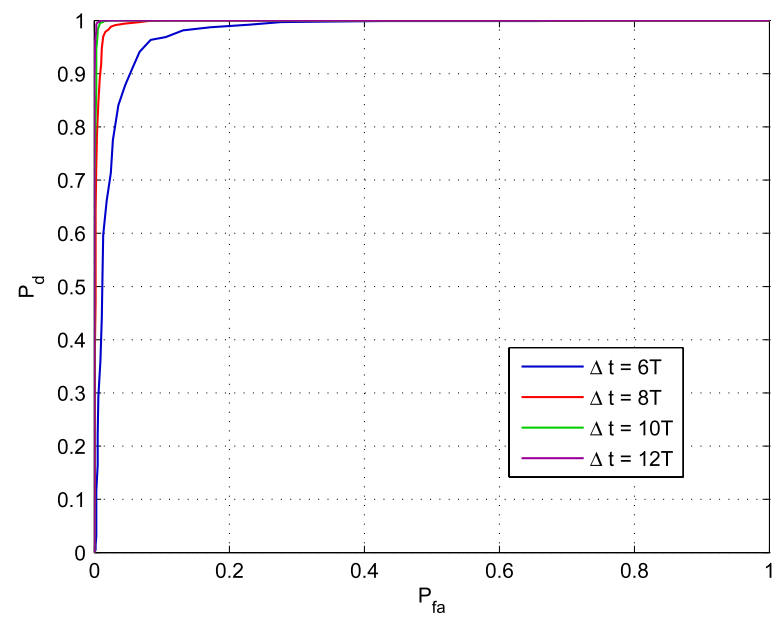

Fig. 8. ROC performance with increasing observation time for $M=5$.

3, 5 and 7 at an arbitrarily chosen cyclic frequency. Gaussian white noise is added for SNR $\approx 5 \mathrm{~dB}$ and a random timing offset is used as in the previous section. Monte Carlo simulations are used to estimate probabilities of detection, $P_{d}$ and false alarm, $P_{f a}$ as determined over 2000 runs. Simulations are repeated using observation times of between 6 and 16 symbol durations, $T$, for each subcarrier set size. Figs. 7-9 illustrate results for subcarrier set sizes of 3, 5 and 7 respectively.

It can be seen that detection performance improves considerably with increasing signal observation time. For a signature generated using 3 mapped subcarriers, an observation time of 16 symbol durations yields a false alarm rate of $1 \%$ for an associated detection rate of $99 \%$ as determined over 2000 simulations. A significant reduction in required observation time may be achieved by adopting a larger mapped subcarrier set size. Indeed Fig. 8 shows that for $M=5$, just 10 symbol durations are required for comparable performance. As can be seen in Fig. 9, this value drops to just 8 symbol durations for $M=7$.

This reduction in required observation time can be explained using the temporal-spectral resolution product. 


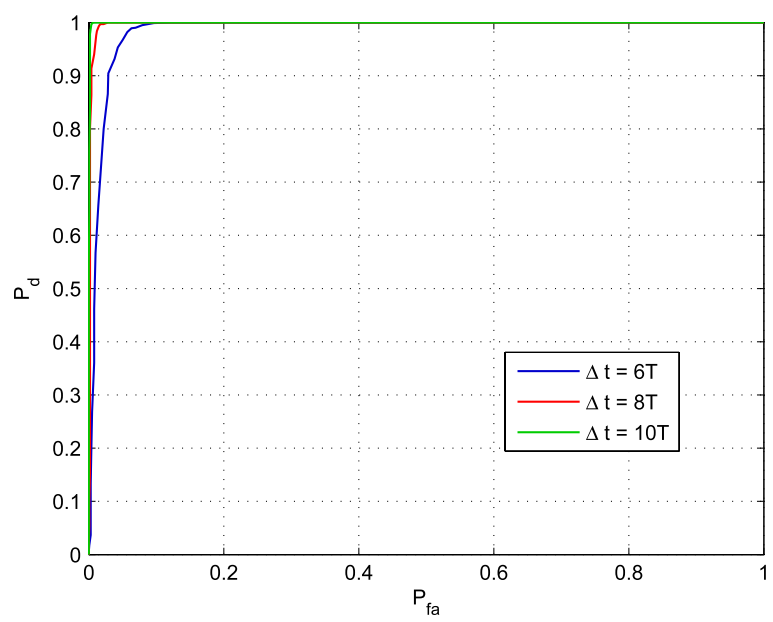

Fig. 9. ROC performance with increasing observation time for $M=7$.

Gardner states that the reliability of an SCF estimate calculated using time or frequency-smoothing depends upon the resolution product [21],

$$
\Delta t \Delta f \gg 1
$$

where $\Delta t$ and $\Delta f$ are the temporal and spectral resolutions respectively. The size of the moving average window used in the signature detector is dictated by the size of the subcarrier set mapped in order to generate a signature. Thus by increasing the subcarrier set size, we increase the size of the moving average window. As the moving average window effectively performs frequency smoothing on our SCF estimate, a greater value of $\Delta f$ is obtained. Accordingly, a smaller temporal resolution, $\Delta t$ is required for an equivalent estimate reliability.

These simulation results illustrate the improvement in detection performance which can be achieved through the adoption of an AF-based detector. Previous results, obtained using a TS-CCP-based detector, showed that an observation time of 60 symbol durations were required to achieve near perfect detection with $M=3$ [7]. The same performance can be achieved by the AF-based detector using an observation time of just 16 symbol durations.

Signature detector performance in frequency selective fading environments can be reduced when a deep fade occurs at the spectral frequency of mapped subcarriers. One approach to overcome this is to increase the frequency diversity of the cyclostationary signature. This may be achieved through use of multiple mapped subcarrier sets in order to generate features at a number of discrete spectral frequencies. Through use of a constant mapping separation, $p$, each feature occurs at a common single cyclic frequency, $\alpha$. In performing detection, additional complexity is not required as each feature may be individually detected using the single feature detector (see Eq. (6)). It should be noted that unique multiplefeature signatures may still be generated through choice of set spacing $p$ to generate a signature at discrete cyclic frequency $\alpha_{\text {sig. }}$. Thus multiple-feature signatures may also be used to achieve unique waveform identification.

A range of simulations are used to examine detection performance using multiple-feature cyclostationary signatures in frequency-selective fading channels.
Table 1

COST 207 typical urban channel model.

\begin{tabular}{lrlcccc}
\hline Relative power $(\mathrm{dB})$ & -3 & 0 & -2 & -6 & -8 & -10 \\
Delay $(\mu \mathrm{s})$ & 0 & 0.2 & 0.5 & 1.6 & 2.3 & 5.0 \\
\hline
\end{tabular}

Table 2

COST 207 bad urban channel model.

\begin{tabular}{lclccrc} 
Relative power $(\mathrm{dB})$ & -2.5 & 0 & -3 & -5 & -2 & -4 \\
Delay $(\mu \mathrm{s})$ & 0 & 0.3 & 1.0 & 1.6 & 5 & 6.6 \\
\hline
\end{tabular}

Table 3

COST 207 rural area channel model.

\begin{tabular}{lcccccc}
\hline Relative power $(\mathrm{dB})$ & 0 & -4 & -8 & -12 & -16 & -20 \\
Delay $(\mu \mathrm{s})$ & 0 & 0.3 & 1.0 & 1.6 & 5 & 6.6 \\
\hline
\end{tabular}

Table 4

COST 207 hilly terrain channel model.

\begin{tabular}{lrrrrrr}
\hline Relative power $(\mathrm{dB})$ & 0 & -1.5 & -4.5 & -7.5 & -8 & -17.7 \\
Delay $(\mu \mathrm{s})$ & 0 & 0.1 & 0.3 & 0.5 & 15 & 17.2 \\
\hline
\end{tabular}

Table 5

Exponential decay channel model.

\begin{tabular}{lrrrrrc}
\hline Relative power $(\mathrm{dB})$ & 0 & -2 & -4 & -6 & -8 & -10 \\
Delay $(\mu \mathrm{s})$ & 0 & 1 & 2 & 3 & 4 & 5 \\
\hline
\end{tabular}

256-subcarrier OFDM signals are considered as before, with subcarriers distributed as follows: 192 data, 55 guard, 8 pilot and 1 DC. Data is randomly generated and QPSK modulated with a 16 sample cyclic prefix prepended to each OFDM symbol. Cyclostationary features are embedded at cyclic frequency $\alpha=16 / T_{s}$ using mapped sets of 3 subcarriers. A $4 \mathrm{MHz}$ signal is simulated with a number of frequency-selective multipath channels modelled using the COST 207 [22] channel profiles as well as an exponentially decayed channel model. Signatures are generated using between 1 and 3 unique features and receiver operating characteristic ( $R O C$ ) performance is examined for each using Monte Carlo simulations. Probabilities of detection $\left(P_{d}\right)$ and false alarm $\left(P_{f a}\right)$ are recorded over 2000 simulations. Gaussian white noise is added for $\mathrm{SNR} \approx 5 \mathrm{~dB}$ and a single feature detector with signal observation time of $\Delta t=30 T$ is used. The delay profiles for each channel model are presented in Tables 1-5 and the ROC performance for each signature type are presented in Figs. 10-11.

Results show the reduction in ROC performance for single feature signatures under frequency-selective fading. For a false alarm rate of 0 as determined over 2000 simulation runs, a single feature signature can achieve an average detection rate of approximately $75 \%$. Using a signature comprising 2 unique features, the detection rate increases to $\approx 92 \%$ when averaged across the channel models, increasing to $\approx 98 \%$ for a 3 -feature signature.

Although improved performance is achieved using multiple feature signatures, these improvements come with the cost of increased overhead.

In order to examine signature detection performance under conditions of mobility, further simulations were 

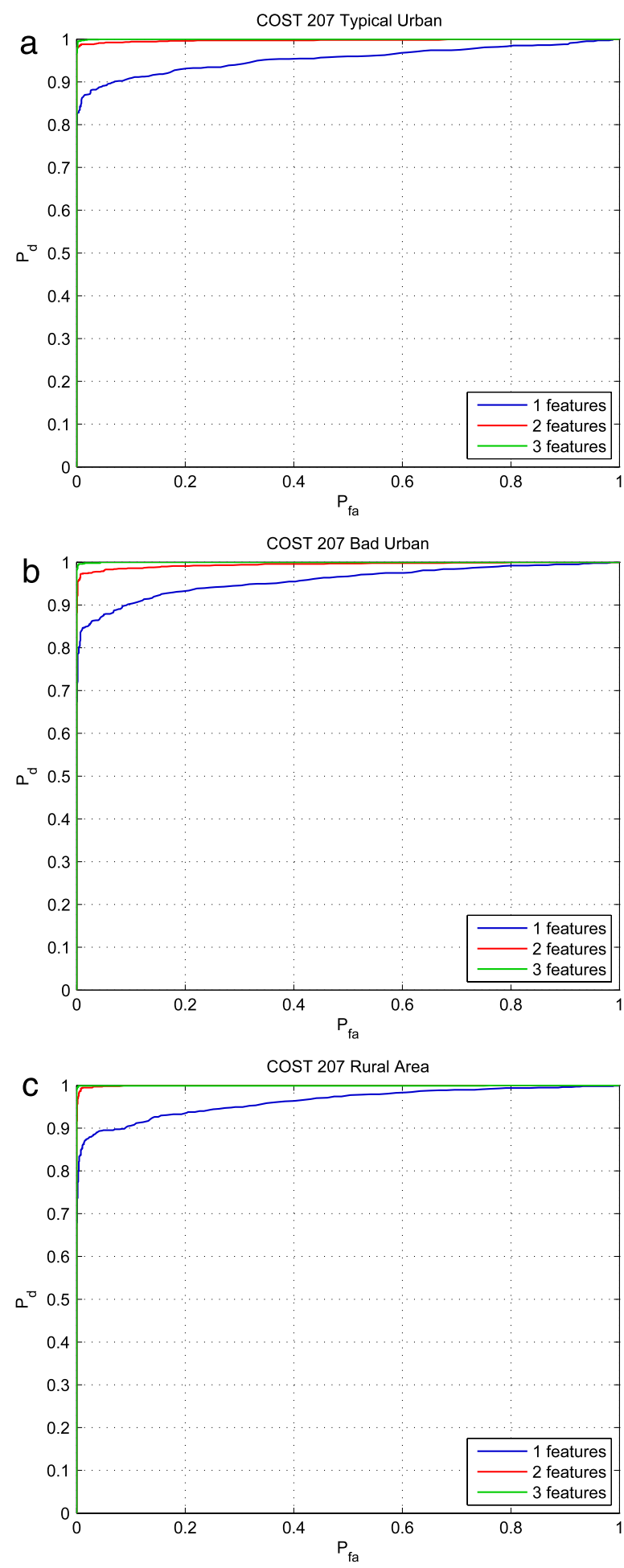

Fig. 10. ROC performance for multiple-feature signatures.

carried out. These simulations assess performance in the presence of both frequency and time-selective fading channels.

256-subcarrier OFDM signals were considered as before, with subcarriers distributed as follows: 192 data, 55
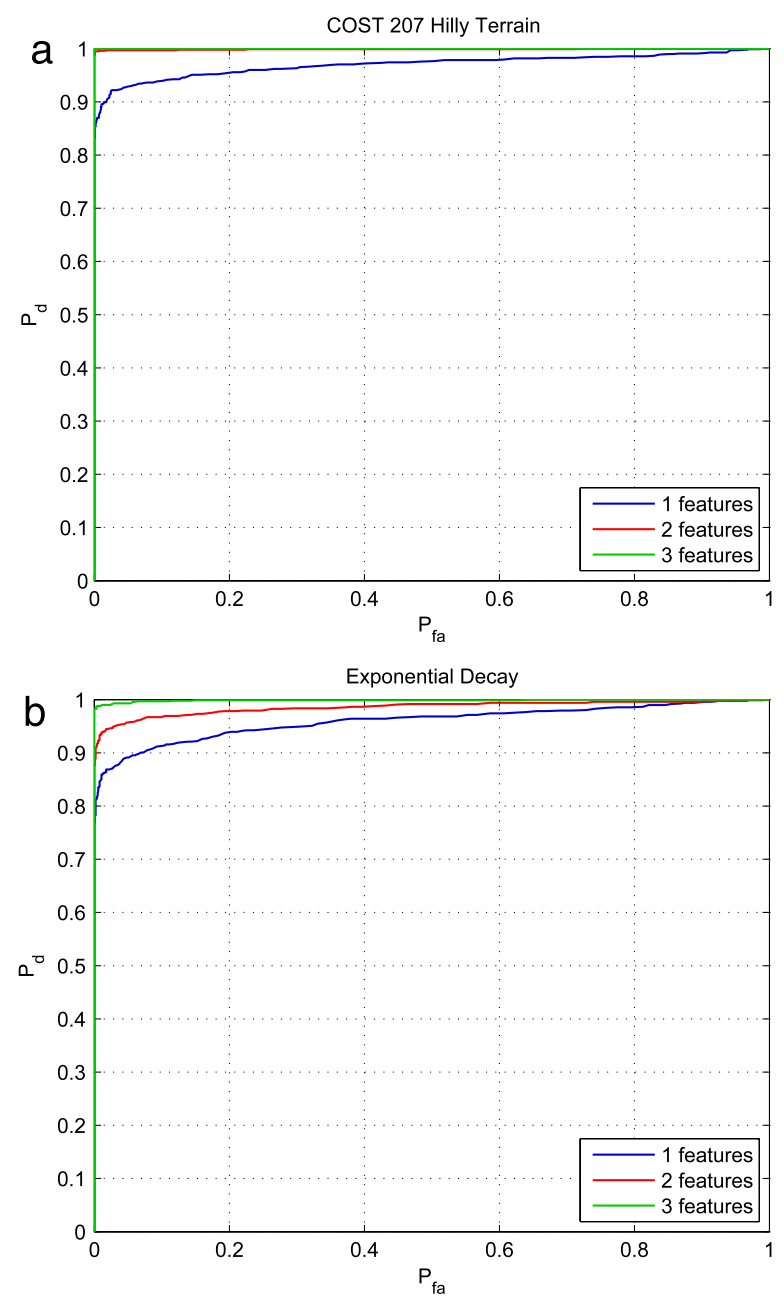

Fig. 11. ROC performance for multiple-feature signatures.

guard, 8 pilot and $1 \mathrm{DC}$. Data was randomly generated and QPSK modulated with a 16 sample cyclic prefix prepended to each OFDM symbol. Cyclostationary features were embedded at cyclic frequency $\alpha=16 / T_{s}$ using mapped sets of 3 subcarriers. A $4 \mathrm{MHz}$ signal was simulated, giving a subcarrier spacing comparable to that used in LTE [23]. An exponentially decayed channel model was chosen as it was shown in the previous section that this was the most challenging model for our detector. Signatures were generated using between 1 and 3 unique features and ROC performance was examined for each using Monte Carlo simulations. Probabilities of detection $\left(P_{d}\right)$ and false alarm $\left(P_{f a}\right)$ were recorded over 2000 simulations. Gaussian white noise was added for SNR $\approx 5 \mathrm{~dB}$ and a single feature detector with signal observation time of $\Delta t=30 T$ was used.

Conditions of fast fading were simulated using the Jakes doppler power spectrum model for a carrier frequency of $2 \mathrm{GHz}$. Maximum doppler shifts were used to simulate velocities of between 25 and $300 \mathrm{~km} / \mathrm{h}$. Results are presented in Figs. 12 and 13.

Results show that cyclostationary signatures can be successfully detected at speeds of up to $300 \mathrm{~km} / \mathrm{h}$ with $P_{d}$ of $91 \%$ for an associated $P_{f a}$ of $10 \%$ using 3 independent embedded features, each generated by mapping 3 subcarriers. 

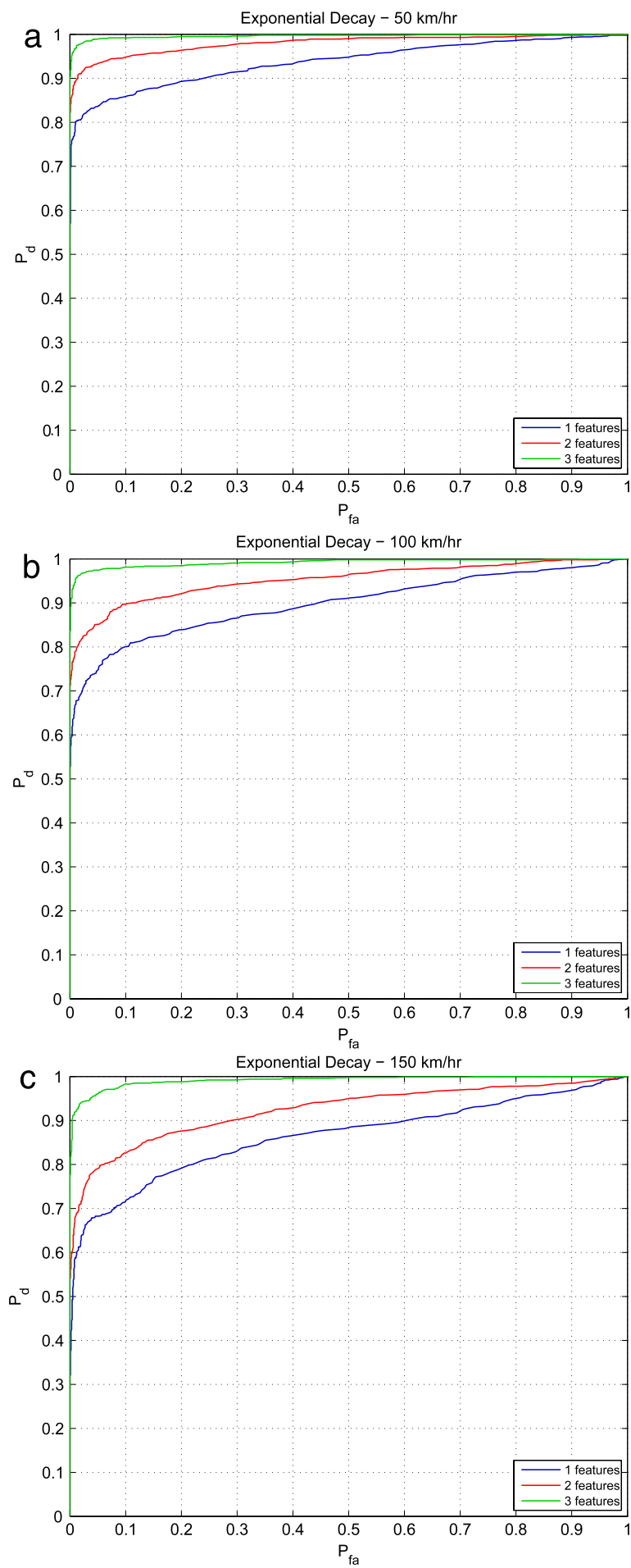

Fig. 12. Signature detection performance under fast fading conditions.

It can be seen in Figs. 12 and 13 that fast fading can deteriorate the performance of our signature detector. This is most clearly seen for a single-feature signature. For an exponentially decayed channel, a single-feature signature can be detected with $P_{d}$ of $90 \%$ for a $P_{f a}$ of $10 \%$. With increasing mobility, this drops to a $P_{d}$ of $80 \%$ for the same
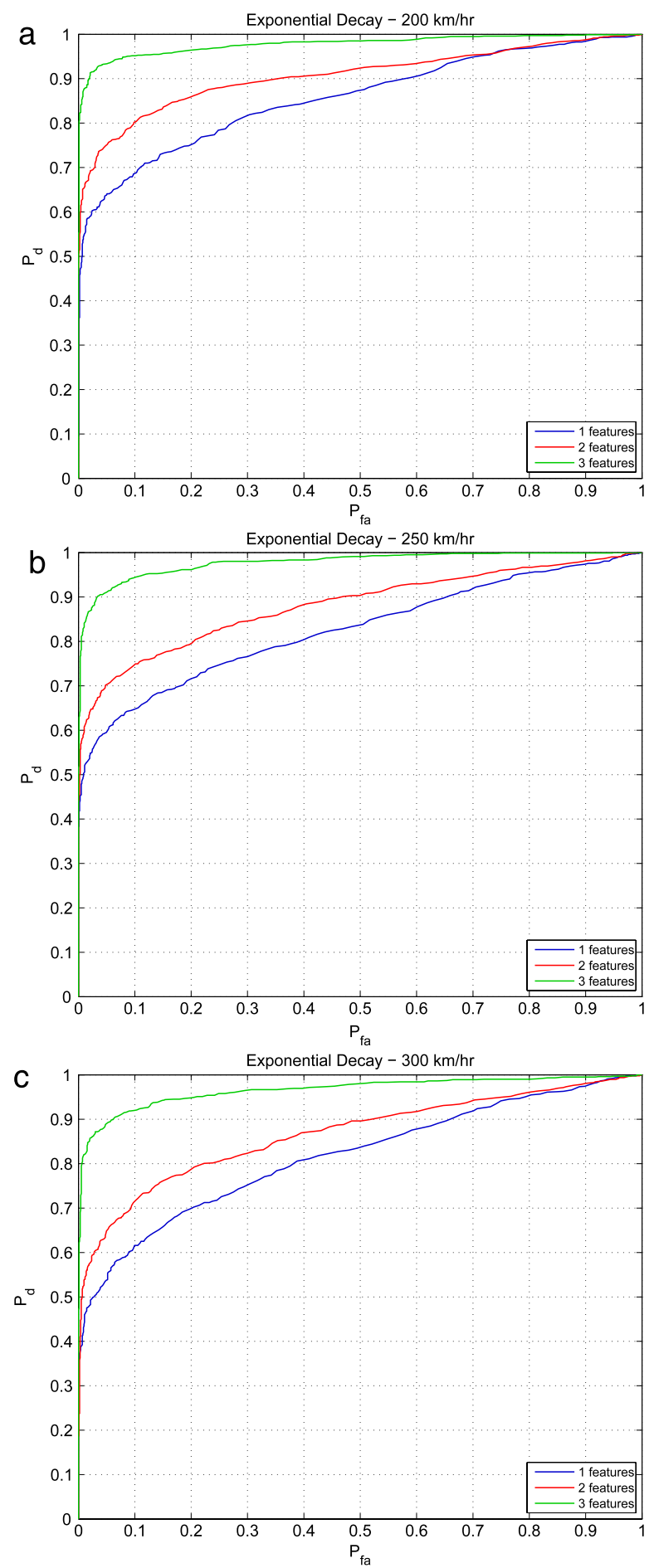

Fig. 13. Signature detection performance under fast fading conditions.

$P_{f a}$ at $100 \mathrm{~km} / \mathrm{h}$. At $300 \mathrm{~km} / \mathrm{h}$ a $P_{d}$ of just $60 \%$ can be achieved for the same $P_{f a}$ using a single-feature signature.

The effect of mobility can however be reduced through use of multiple-feature signatures. Results show that a 3 -feature signature can be detected almost perfectly in an exponentially decayed channel without mobility. At $100 \mathrm{~km} / \mathrm{h}$ a $P_{d}$ of $90 \%$ can be achieved for a $P_{f a}$ of $3 \%$ and at 
$300 \mathrm{~km} / \mathrm{h}$ a $P_{d}$ of $91 \%$ can be achieved for an associated $P_{f a}$ of $10 \%$. For our example, where a 256 -subcarrier waveform is used with 192 data carriers, this signature requires a data overhead of less than $5 \%$.

\subsection{Experimentation}

In order to examine the performance of our cyclostationary signature detector using over-the-air signals, an MG3700A vector signal generator was used together with a Universal Software Radio Peripheral (USRP) radio frequency (RF)-front end and a transceiver implemented using the Iris software radio architecture [24]. Suitable signals were generated using the Iris transmitter and stored to file in in-phase-quadrature $(I-Q)$ format. These were then loaded onto the signal generator and transmitted at $2.35 \mathrm{GHz}$ using a test licence. OFDM signals were generated with 256 subcarriers, of which 55 were reserved as guard carriers and 1 was designated the zero-frequency, direct current (DC) carrier. A 1/16 cyclic prefix was added to each symbol generated. Subcarriers were modulated using randomly generated QPSK data symbols and subcarrier set mapping was used to embed single-feature signatures with a cyclic frequency arbitrarily chosen as $\alpha=32 / T_{s}$. Subcarrier set sizes between 1 and 9 were employed to facilitate performance comparisons. Signals without embedded signatures were also generated.

Generated signals were transmitted with a sample rate of $1 \mathrm{MHz}$ using the signal generator with centre frequency $2.35 \mathrm{GHz}$. These transmitted signals were captured at a distance of $3 \mathrm{~m}$ using an RFX2400 USRP daughterboard set to sample a bandwidth of $1 \mathrm{MHz}$ centred at $2.35 \mathrm{GHz}$. The downconverted samples were then transferred to the Iris software radio engine via USB and processed by the receiver. Transmit power levels were set between -8 and $-36 \mathrm{~dB} \mathrm{~m}$ to give an estimated receive SNR of between 12 and $-6 \mathrm{~dB}$.

SNR estimation at the receiver was achieved using captured signals comprising noise only and both noise and a known OFDM signal. The power spectral density (PSD) was calculated for each and averaged over 1000 windows of 1024 samples. Mean powers were estimated over bandwidths of both the noise only $\left(P_{n}\right)$ and the noise and signal samples $\left(P_{s+n}\right)$. These mean powers were then used to calculate the estimated SNR as:

$\mathrm{SNR}_{\text {est }}=10 \log _{10}\left(\frac{P_{s+n}}{P_{n}}-1\right)$.

Within the receiver, the signature detector was used to calculate the detection statistic, $y_{\alpha}$ for a signal observation time equivalent to 30 OFDM symbol durations, $\Delta t=$ $30 T$. 1000 runs were used to calculate average detection statistics for signals both with and without signatures. A detection ratio $y_{\text {sig }} / y_{0}$ was calculated using the same approach as that taken in our simulations (see Section 4.1).

Experimental results are illustrated in Fig. 14 for estimated SNR between -6 and $12 \mathrm{~dB}$. Equivalent results obtained using simulations are illustrated in Fig. 6 in the previous section and a direct comparison for a subset of results is illustrated in Fig. 15. Here, experimental results are illustrated using continuous lines and simulation results

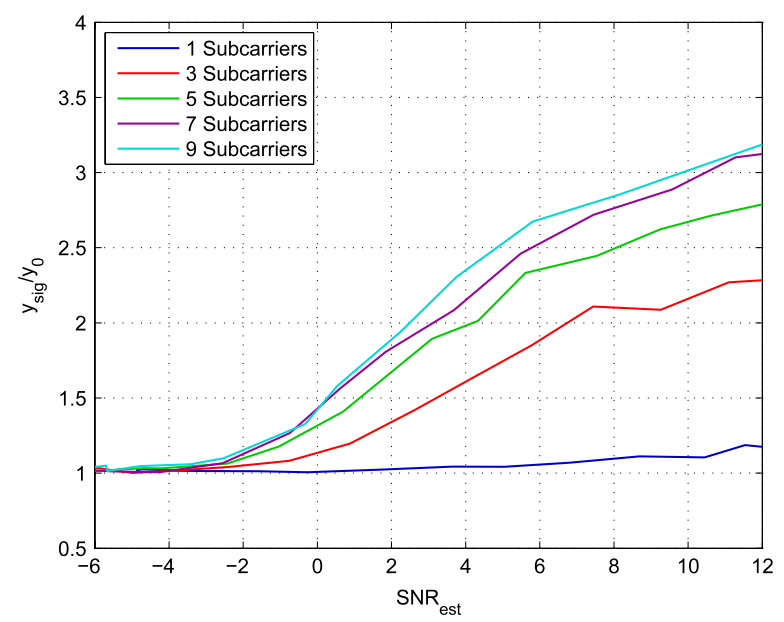

Fig. 14. Signature detection ratio performance with increasing mapped subcarrier set sizes over a range of Signal to Noise Ratio (SNR) values.

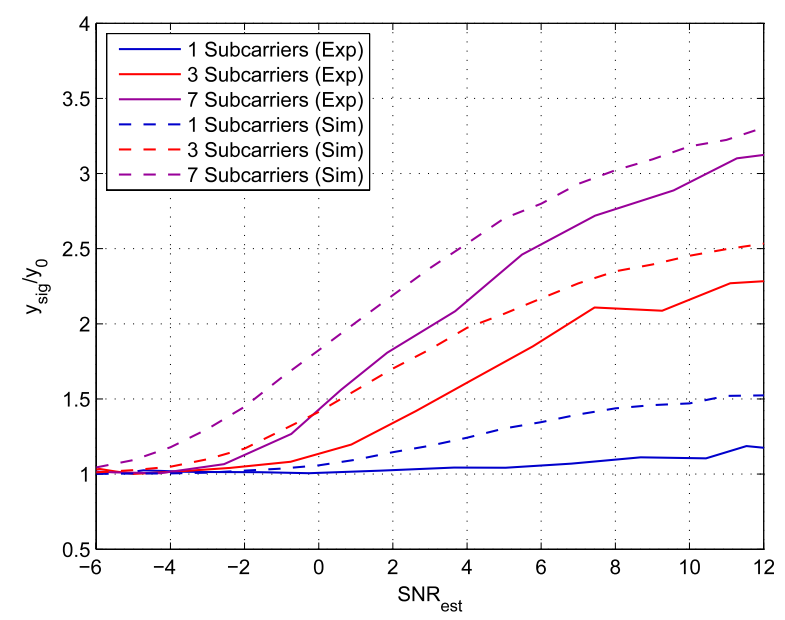

Fig. 15. Comparison of simulation and experimental results for signature detection.

are illustrated using dashed lines. Comparing these results, it can be seen that a similar improvement in detection performance is observed with increasing subcarrier set size. While a detection ratio of $\approx 1.5$ can be achieved using a set of 3 subcarriers for an estimated SNR of $3 \mathrm{~dB}$, this ratio increases to $\approx 2$ when a subcarrier set size of 7 is employed. In terms of absolute ratio values however, experimental results exhibit a decrease in performance over those obtained through simulation. For example, at $5 \mathrm{~dB}$ SNR, a 3-subcarrier signature is detected with ratio $y_{\text {sig }} / y_{0} \approx 2$ using simulations. This value falls to $\approx 1.75$ for experimental results. An explanation for this fall in performance is the use of white Gaussian noise in simulations. Under experimental conditions, the wireless channel noise is unlikely to be perfectly white and uncorrelated, resulting in the reduced performance observed. Although detector performance falls slightly under experimental conditions, cyclostationary signatures may be used to achieve excellent detection results for signals received with SNR of greater than $0 \mathrm{~dB}$. 


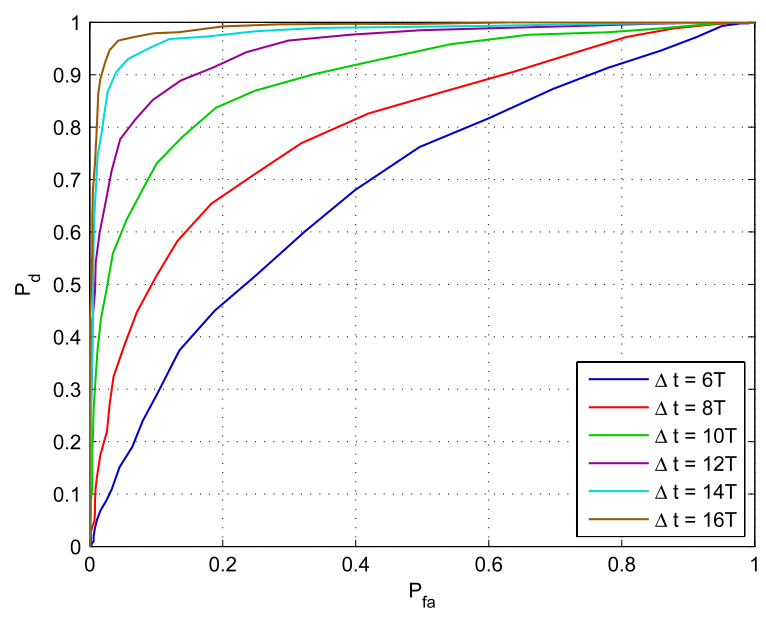

Fig. 16. ROC performance with increasing observation time for $M=3$.

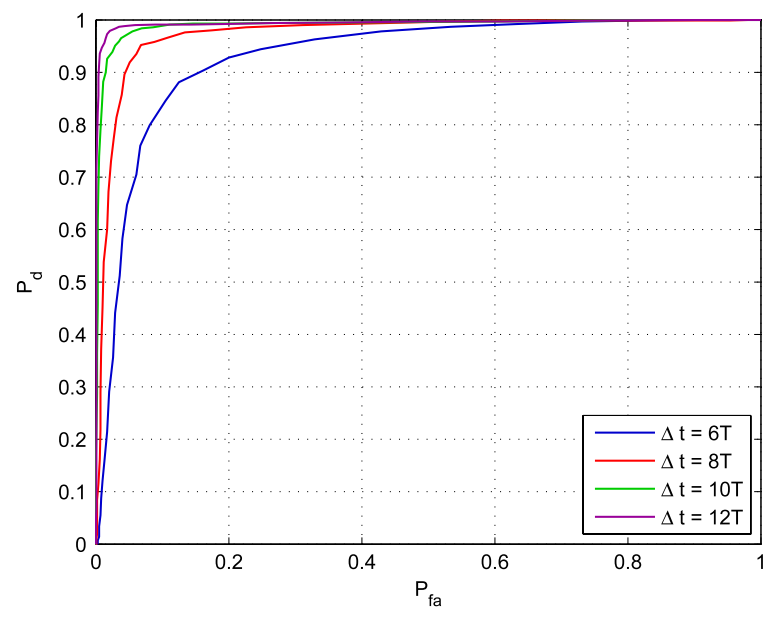

Fig. 17. ROC performance with increasing observation time for $M=5$.

In generating a cyclostationary signature, mapped subcarrier set sizes are the key parameter in determining the detection performance which can be achieved. However, in detecting an embedded signature, the observation time employed in the detector is the key factor.

Experiments were carried out using OFDM signals as before. Suitable signals were generated and transmitted using the MG3700A signal generator. Single feature signatures were embedded in a number of signals using mapped subcarrier set sizes of 3,5 and 7. A transmit power of $-16 \mathrm{~dB} m$ was used to give an estimated receive SNR of $\approx 5 \mathrm{~dB}$. Detection statistics were recorded over a range of observation times for signals with and without signatures and used to generate the ROC graphs illustrated in Figs. 16-18.

Results show that significant improvements in detection performance can be achieved using increased observation times. Using a 3-subcarrier signature, a detection rate of $90 \%$ with an associated false alarm rate of $15 \%$ can be achieved using an observation time equivalent to 12 OFDM symbol durations. By increasing the observation time to 16 symbol durations, the achieved detection rate increases to $\approx 98 \%$ with an associated false alarm rate of $\approx 2 \%$. Figs. 17

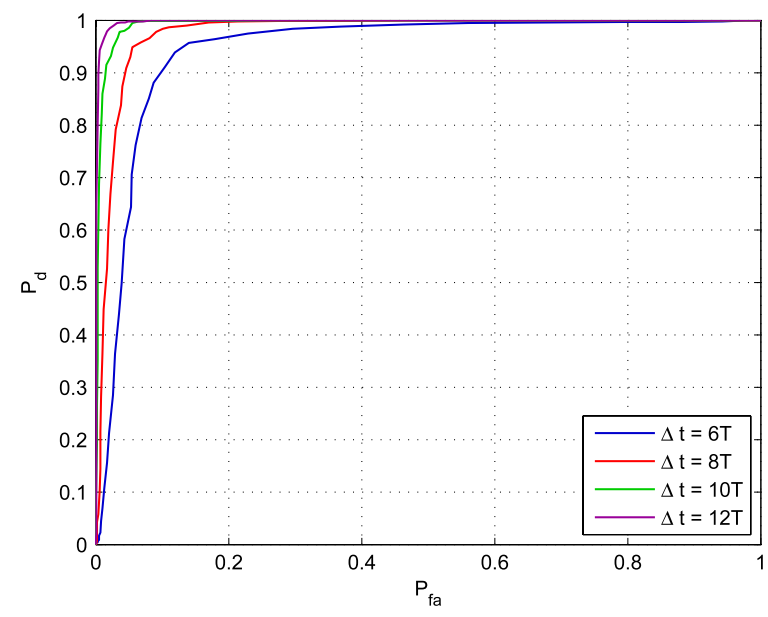

Fig. 18. ROC performance with increasing observation time for $M=7$.

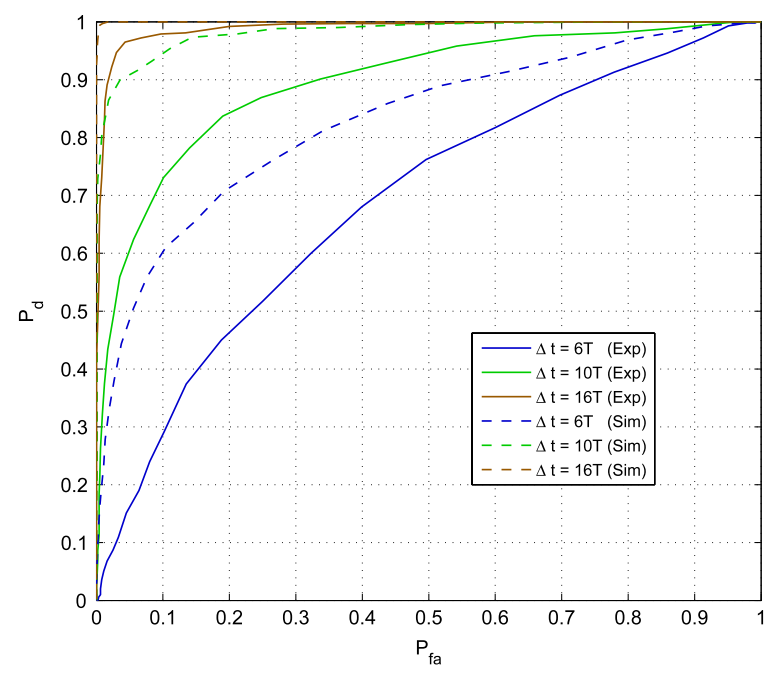

Fig. 19. Comparison of simulated and experimental ROC performance with increasing observation time for $M=3$.

and 18 show that comparable performance can be achieved using reduced observation times for signatures generated with greater subcarrier set sizes.

Fig. 19 directly compares experimental results for $M=$ 3 with those obtained using simulations in Section 4.1. Comparisons show a fall in detector performance for realworld experiments. Similarly to the fall in performance observed in the previous section, this can be explained by the use of white Gaussian noise in simulations. As realworld channels rarely exhibit perfectly white, uncorrelated noise, this can reduce the reliability of our SCF estimates.

\section{Conclusions}

This paper has presented cyclostationary signatures as a powerful tool for overcoming the challenge of network rendezvous and coordinations in LTE Advanced networks and beyond. Two mechanisms for embedding signatures in downlink LTE CC waveforms were examined and the 
relative benefits and drawbacks of each were discussed. A signature detector based on the autocoherence function was described and its performance was examined through both simulation and experiment. It was seen that signatures can be robustly detected under conditions of severe time and frequency-selective fading and performance improvements achieved by this detector design were highlighted in terms of required observation times.

\section{Acknowledgement}

This material is based upon work supported by Science Foundation Ireland under Grant No. 10/CE/I853 as part of CTVR at University of Dublin, Trinity College, Ireland.

\section{References}

[1] 3GPP TR 36.814 v. 9.0.0, Further advancements for E-UTRA physical layer aspects, Technical Specification Group Radio Access Network, Rel. 9, 2010.

[2] 3GPP TS 36.104 v. 10.3.0, Base station (BS) radio transmission and reception, Technical Specification Group Radio Access Network, Rel. $10,2011$.

[3] Federal Communications Commission, Second report and order and memorandum opinion and order in the matter of unlicensed operation in the tv broadcast bands and additional spectrum for unlicensed devices below $900 \mathrm{MHz}$ and in the $3 \mathrm{GHz}$ band (14.11.2008).

[4] T. Forde, L. Doyle, B. Özgül, Dynamic block-edge masks (BEMs) for dynamic spectrum emission masks (SEMs), in: 4th IEEE Symposium on New Frontiers in Dynamic Spectrum Access Networks, 2010. DySPAN 2010, 2010.

[5] P.D. Sutton, K.E. Nolan, L.E. Doyle, Cyclostationary signatures for rendezvous in ofdm-based dynamic spectrum access networks, in: 2nd IEEE International Symposium on, New Frontiers in Dynamic Spectrum Access Networks, 2007. DySPAN 2007, 2007, pp. 220-231. http://dx.doi.org/10.1109/DYSPAN.2007.37.

[6] P. Sutton, J. Lotze, K. Nolan, L. Doyle, Cyclostationary signature detection in multipath Rayleigh fading environments, in: 2nd International Conference on Cognitive Radio Oriented Wireless Networks and Communications, 2007.

[7] P.D. Sutton, K.E. Nolan, L.E. Doyle, Cyclostationary signatures in practical cognitive radio applications, IEEE J. Sel. Areas Commun. 26 (1) (2008) 13-24.

[8] P.D. Sutton, B. Özgül, K.E. Nolan, L.E. Doyle, Bandwidth-adaptive waveforms for dynamic spectrum access networks, in: IEEE International Symposium on New Frontiers in Dynamic Spectrum Access Networks, DySPAN, Chicago, IL, 2008, pp. 1-7.

[9] B. Ozgul, P. Sutton, L. Doyle, Bandwidth-adaptive MIMO OFDM for dynamic spectrum access networks, Phys. Commun. 2 (3) (2009) 194-203. http://dx.doi.org/10.1016/j.phycom.2009.01.002.

[10] W.A. Gardner, Cyclostationarity in Communications and Signal Processing, IEEE Press, New Jersey, NY, USA, 1993.

[11] W.A. Gardner, Signal interception: a unifying theoretical framework for feature detection, IEEE Trans. Commun. 36 (1988) 897-906.

[12] A. Fehske, J. Gaeddert, J. Reed, A new approach to signal classification using spectral correlation and neural networks, in: 2005 First IEEE International Symposium on, New Frontiers in Dynamic Spectrum Access Networks, 2005. DySPAN 2005. 2005, pp. 144-150. http://dx.doi.org/10.1109/DYSPAN.2005.1542629.

[13] F. Gini, G. Giannakis, Frequency offset and symbol timing recovery in flat-fading channels: a cyclostationary approach, IEEE Trans. Commun. 46 (3) (1998) 400-411.

[14] H. Bolcskei, Blind estimation of symbol timing and carrier frequency offset in wireless OFDM systems, IEEE Trans. Commun. 49 (6) (2001) 988-999.

[15] M. Tsatsanis, G. Giannakis, Transmitter induced cyclostationarity for blind channel equalization, IEEE Trans. Signal Process. 45 (7) (1997) 1785-1794 (see also IEEE Transactions on Acoustics, Speech, and Signal Processing)

[16] D. Vučić, M. Obradović, D. Obradović, Spectral correlation of OFDM signals related to their PLC applications, in: 6th International Symposium on Power-Line Communications and its Applications, ISMPLC, 2002.

[17] B. Sadler, A. Dandawate, Nonparametric estimation of the cyclic cross spectrum, IEEE Trans. Inform. Theory 44 (1) (1998) 351-358. http://dx.doi.org/10.1109/18.651065.
[18] W. Gardner, Introduction to Random Processes with Applications to Signals and Systems, MacMillan, 1985.

[19] ITU-R, Framework and overall objectives of the future development of imt-2000 and systems beyond imt-2000, M.1645 (06/03), June 2003.

[20] H. Harada, R. Prasad, Simulation and Software Radio for Mobile Communications, Artech House, 2002.

[21] W. Gardner, Measurement of spectral correlation, IEEE Trans. Acoust. Speech Signal Process. 34 (5) (1986) 1111-1123 (see also IEEE Transactions on Signal Processing).

[22] M. Failli, (chairman) and COST 207 Management Committee, Digital Land Mobile Radio Communications: Final Report, Commission of the European Communities, Luxembourg, 1989.

[23] J. Zyren, Overview of the 3GPP Long Term Evolution Physical Layer, Freescale White Paper, July 2007.

[24] P.D. Sutton, J. Lotze, H. Lahlou, S.A. Fahmy, K.E. Nolan, B. Özgül, T.W. Rondeau, J. Noguera, L.E. Doyle, Iris: an architecture for cognitive radio networking testbeds, IEEE Commun. Mag. 48 (9) (2010) 114-122. http://dx.doi.org/10.1109/MCOM.2010.5560595.

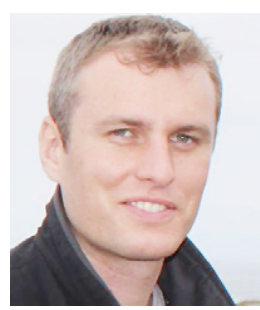

Paul Sutton is the founder of Software Radio Systems Ltd., based in Cork and Dublin, Ireland. He is a senior research fellow at CTVR, the Telecommunications Research Centre, based in Trinity College Dublin and has been actively involved in the research and development of emerging wireless technologies since 2004. In this time, his work has addressed key challenges in the areas of dynamic spectrum access and cognitive radio through the use of technologies including cyclostationary signal analysis, adaptive multicarrier waveforms and software radio. Paul served on the board of the Wireless Innovation Forum from 2009 to 2013. Through the design and implementation of wireless baseband processing algorithms on a wide range of processing platforms, Paul has gained experience in developing high-performance highly-parallel software architectures. He has built on this experience as a senior solution architect at Xcelerit, an Irish company specializing in algorithm acceleration for the financial industry.

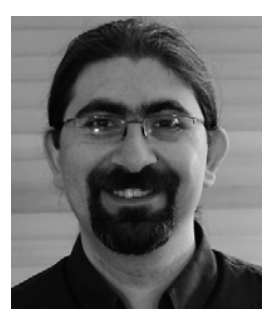

Barıș Özgül received the B.S., M.S. and Ph.D. degrees in electrical and electronics engineering from Boğaziçi University, Turkey, in 1998, 2002 and 2008, respectively. From 1998 to 2003, he worked in Nortel Netaș as a R\&D engineer and software architect. He joined Turkcell in 2003 and worked as an expert in the network platform development team until June, 2005. From June 2005 to March 2008, he was with the Wireless Communications Laboratory at Boğaziçi University. From May 2006 to November 2006, he was a visiting researcher in the Wireless Systems Laboratory, Georgia Institute of Technology. In 2008 he joined CTVR, Trinity College Dublin, as a postdoctoral researcher. He contributed to this paper while he was with CTVR. His research interests in CTVR included wireless communications and next generation wireless network technologies. Since September 2011 he has been with the Xilinx Research Labs, Dublin, Ireland.

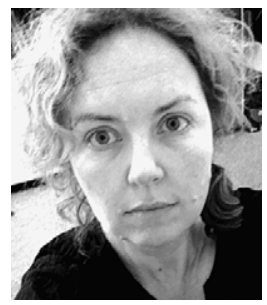

Linda Doyle is director of CTVR/The Telecommunications Research Centre and a faculty member in the School of Engineering in Trinity College, University of Dublin, Ireland. CTVR is the national telecommunications research centre in Ireland, involving six other academic institutions and over 120 researchers. The centre focuses on research in the area of optical and wireless networks and works closely with industry. Linda's areas of expertise are in the domains of cognitive radio, reconfigurable networks, spectrum management and art \& technology. Linda is a member of the Ofcom Spectrum Advisory Board. She is a Fellow of Trinity College Dublin. She is a Director of Xcelerit and SRS, two recent CTVR spin-outs. 